\title{
FUTURE OPPORTUNITIES ARISING FROM IMPLICATIONS OF MOORE'S LAW
}

\author{
Mildred S Dresselhaus \\ Massachusetts Institute of Technology, Cambridge, Massachusetts, USA
}

The semiconductor device world has been fueled for the past 60 or more years by Moore's Law and its implications on semiconductor device technologies, by driving industry to develop eversmaller gate sizes on an annual basis, accompanied by corresponding increased performance of the associated electronics. On one hand, it has been obvious for many years that a limit to Moore's law is inevitable, when the gate size reaches the single molecule length scale. At present we are reaching the 10-nm length scale, so that discussions of the end of Moore's law are becoming more widespread, active, and serious in the nano electronics sector and at international conferences involving nanoscale materials and applications.

Concurrently, electronic devices continue to increase in functionality and energy efficiency. New start-up companies continue to be launched and young people at universities continue to be attracted to this research field and to commit much of their effort into coupling advances in hardware with computer science into start-up companies in the electronics industry. Creativity and inventiveness remain at center stage.

Silicon, along with carbon, is in Column IV of the period table, and is an Earth-abundant material that has made a big mark on modern electronics. Even though new materials are finding niche applications in modern device technology, silicon is expected to remain the material of choice to support much of industrial semiconductor device technology for the foreseeable future, because of its special electronic properties, and because of the huge effort and investment that industry has already made to achieve silicon's present key role in present-day electronics.

However, the research community has had to put significant effort into planning for the future and particularly to what happens when Moore's law comes to an end. This has become an increasingly important issue for public discussion at conferences and in the research literature for the past decade. Scientific progress has in recent years been made faster and faster and planning for the future has been more organized. Combining this societal aspect with activities occurring in organizations and sponsored by governments, studies of research opportunities in the next decade have become more common and have had more impact.

Historically, what has happened in the past when a particular technology stops meeting societal expectations for advance is that competing emergent technologies get a chance to blossom and major advances in technology occur and the standard of living rises as a result. This is what happened with the adoption of electricity as a general energy source just before we entered the 1900s, soon followed by the adoption of AC rather than DC power, and after several decades the appearance of computers and the start of the digital world.

Several new research directions have already emerged as paving the way for reaching center stage when the era of Moore's law, as we know it, ends. Two clear developments that have clearly taken hold is the development of new research directions for electronics research, along with the discovery of new science associated with the new materials. Firstly, in the United States, the funding agencies have been very helpful in organizing studies with wide participation motivated by the research community through the decadal studies by the National Academies of Science and Engineering, which were effectively extended by more detailed studies organized by the Department of Energy's Office of Science and other governmental agencies and implemented by providing group grants to promote collaborative interdisciplinary studies. In Europe, there were regional and topical programs, such as the flagship program on graphene and nanoscience which was soon extended to other popular layered materials with bandgaps. Individual counties also were motivated to start local programs around a theme and these countries started to award group grants promoting collaborative teams to solve larger scale scientific problems using teamwork approaches. The approaches in time became more popular following international trends.

Now let me address some specifics about my own research field in quite general terms. In the field of materials physics, threats of bringing Moore's law to smaller and smaller length scales has resulted in a revolution supporting the research on new materials that had not been much studied before at the nanoscale and the study of the new science brought by the studies of these materials.

The new materials under study nowadays fall into different categories, which can be classified in different ways, such as their dimensionally, their special properties, their method of preparation, their potential practical use, their potential scientific use, and their potential in serving as a standard reference material. Let us now consider some of these categories further.

Under the category of dimensionality, in the past my group considered bulk or three-dimensional (3D) materials almost exclusively, since these were the only materials that we could then make in the laboratory. We, of course, have considered lower-dimensionality materials abstractly in our imagination since the beginning of my independent career in1960, and we studied low-dimensional materials to some extent in actuality. I for one was attracted to study carbon-based materials because they were abundant on our planet and they had properties different than silicon, which was a highly popular material. I recognized in my early career that silicon along with carbon are both in Column IV of the period table, and are Earthabundant materials. Silicon, however, had made a big mark on modern electronics and carbon had not in my early career. Even though new materials are finding niche applications in modern device technology, silicon is expected to remain the material of choice to support much of industrial semiconductor device technology for the foreseeable future, because of its special electronic properties, and because of the huge effort and investment that industry has already made to achieve its present key role in present-day electronics.

In 1992 my group wrote a paper saying that it would be interesting to study carbon nanotubes because they were a new type of one-dimensional-like material. Without changing its chemical composition, carbon could be either semiconducting or metallic in the form of single-wall nanotubes, depending on their chirality. Interestingly, it took six years more before we learned how to prepare carbon nanotubes well enough to verify that this concept was indeed correct, but once the preparation method was demonstrated in a simple way in our 1998 paper, it soon became easy enough to demonstrate by high school students in their own classrooms.

Another characteristic of nanoscale materials like carbon nanotubes is that they can be doped with certain chemical species to give them other interesting properties, such as various types of magnetism or superconductivity which would be different from the properties of their bulk counterparts relative to critical field, and critical temperature. 
Yet another type of different property of the low-dimensional form of carbon relative to its bulk counterpart would be in the formation of nanotube ensembles whereby the nanotubes could be aligned, showing different properties from the individual tubes, with different properties found if all the tubes in the ensemble were of the same chirality, or were all metallic or all semiconducting. Different properties could also be achieved by ensembles of ordered vs. disordered nanotubes.

Almost every category of property mentioned above has had some scientific interest from a metrological viewpoint, and the metrology of low-dimensional systems is a topic of current interest that has entered the intellectual property world with products associated with some of them. The simple $(5,4)$ semiconducting nanotube may become a simple standard because people can easily make it and use it for semiconducting applications.
Nanotubes are 1D systems that are likely to depend on their preparation method, and materials synthesis and preparation are other topics worthy of discussion relative to what may happen in the era of beyond Moore's law. In recent years the nanoscience research community increased rapidly. Carbon nanotubes became popular in the early 1990s, but the associated research community and the resulting commercial world kept growing, especially after the discovery of graphene in 2004, which fueled another large expansion ready to exploit the new layered materials now under development. These new materials include nanoscale forms of transition metal dichalcogenides of many kind with a large variety of properties, which are getting ready for exploitation by the next wave of young people looking for new opportunities in the nanoworld.

\section{CONTACT}

Mildred S. Dresselhaus, tel +1-617-253-6864; millie@mgm.mit.edu 